\title{
Endoluminal Cooling of the Esophagus during Percutaneous Thermal Ablation for Juxtaesophageal Liver Neoplasm: Two Cases
}

\author{
Chihiro Itou ${ }^{1}$ Yasuaki Arai ${ }^{1} \quad$ Miyuki Sone $^{1} \quad$ Shunsuke Sugawara $^{1}$ \\ ${ }^{1}$ Department of Diagnostic Radiology, National Cancer Center \\ Hospital, Chuo-ku, Tokyo, Japan \\ Address for correspondence Chihiro Itou, MD, PhD, Department of \\ Diagnostic Radiology, National Cancer Center Hospital, 5-1-1 Tsukiji, \\ Chuo-ku, Tokyo 104-0045, Japan (e-mail: chitou@ncc.go.jp).
}

Arab J Intervent Radiol 2021;5:48-51.

\begin{abstract}
Keywords

- endoluminal irrigation

- esophagus

- liver neoplasm

- nasal feeding tube

- thermal ablation
\end{abstract}

\section{Introduction}

Esophageal injury associated with ablation therapy for liver neoplasm is rare, ${ }^{1-3}$ and esophageal protection has not been emphasized despite the increasing use of thermal ablation with the development of adjunctive thermoprotection. ${ }^{4}$ However, esophageal perforation with delayed stenosis was reported as a severe complication related to liver ablation, even with laparoscopic mobilization. ${ }^{5}$

Here, we report two consecutive cases of juxtaesophageal liver tumors that were successfully treated with thermal ablation, using continual intraluminal irrigation with cooled saline via a nasal feeding tube placed into the lower esophagus during the procedure.

\section{Case 1}

A 82-year-old man underwent partial hepatectomy for hepatocellular carcinomas (HCCs), followed by radiofrequency ablation (RFA) for another HCC 5 years later. However, another solitary HCC developed in segment II, just below the diaphragm 1 year after the last treatment. This tumor was very close to the lower esophagus ( - Fig. 1) Microwave ablation (MWA) was preferred to RFA for the locoregional treatment because the tumor was $30 \mathrm{~mm}$ in diameter and also abutted the left hepatic vein.

Following transarterial chemoembolization with epirubicin-lipiodol emulsion plus gelatin sponge particle on day 1 , MWA was performed on day 2 using the Emprint Ablation published online June 4, 2021
DOI https://doi.org/

$10.1055 / \mathrm{s}-0041-1730119$ ISSN 2542-7075 (c) 2021. The Pan Arab Interventional Radiology Society

This is an open access article published by Thieme under the terms of the Creative Commons Attribution-NonDerivative-NonCommercial-License, permitting copying and reproduction so long as the original work is given appropriate credit. Contents may not be used for commercial purposes, or adapted, remixed, transformed or built upon. (https://creativecommons.org/licenses/by-nc-nd/4.0/).

Thieme Medical and Scientific Publishers Private Ltd. A-12, Second Floor, Sector -2, NOIDA -201301, India 


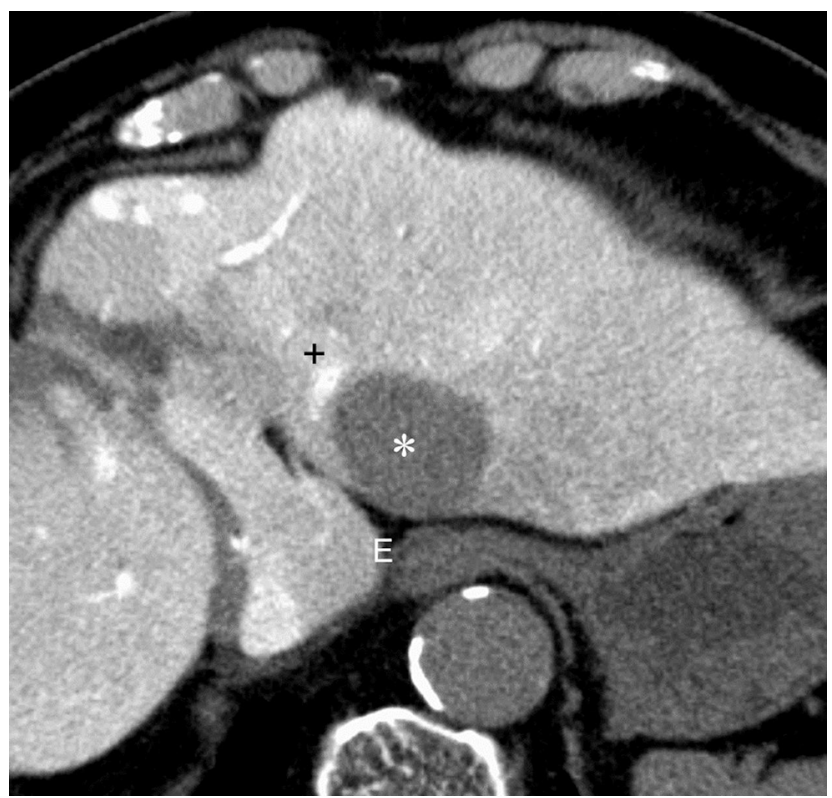

Fig. 1 Computed tomography during arterial portography depicts a 30-mm hepatocellular carcinoma in segment II as a well-demarcated contrast defect $\left({ }^{*}\right)$ abutting both the lower esophagus (E) and the left hepatic vein $(+)$.

System with Thermosphere Technology (Covidien Japan, Tokyo, Japan) under continuous intravenous sedation with propofol combined with intermittent fentanyl administration and local anesthesia with lidocaine. Because of the juxtacardiac location of the tumor, artificial ascites was created to protect the diaphragm, followed by the placement of a 12-French nasal feeding tube with side holes (Create Medic, Kanagawa, Japan) into the lower esophagus. Under fluoroscopic guidance, a single antenna was subsequently inserted into the tumor, which was clearly visible as an area of dense lipiodol accumulation ( - Fig. 2). After the correct placement of the antenna was confirmed on intraprocedural computed tomography (CT) ( - Fig. 3], MWA was performed at $100 \mathrm{~W}$ for 10 minutes. During the ablation, repeated irrigation was performed with a $30 \mathrm{~mL}$ saline cooled to $6^{\circ} \mathrm{C}$ at a speed of 15 to $20 \mathrm{~mL}$ per minute, followed by aspiration via the tube. The tube was withdrawn after the ablation was completed. The day after the procedure, the patient developed moderate pain at the puncture site and fever exceeding $38^{\circ} \mathrm{C}$, requiring an analgesic with antipyretic properties. Similarly, the patient suffered from a loss of appetite for several days that subsided gradually, and he was discharged 6 days after the procedure.

Contrast-enhanced CT obtained 14 days after the treatment showed no esophageal injury ( - Fig. 4). No local recurrence nor delayed esophageal complications were observed on imaging evaluations performed 15 months after the treatment.

\section{Case 2}

A 20-year-old female patient who underwent gastrectomy for gastrointestinal stromal tumor had multiple metachronous liver metastases, including a $12-\mathrm{mm}$ tumor located

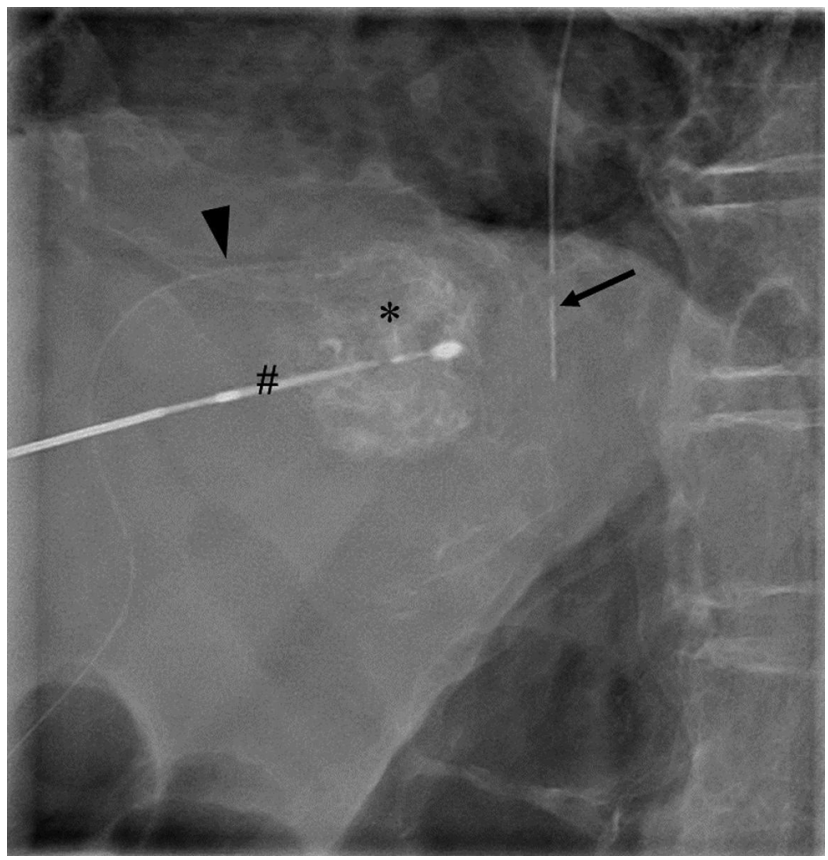

Fig. 2 A microwave ablation antenna (\#) is inserted in the well-opacified tumor $\left({ }^{*}\right)$ with lipiodol accumulation following placement of a nasal feeding tube (arrow) into the lower esophagus, and a 6-French tube (arrowhead) below the diaphragm to create artificial ascites.

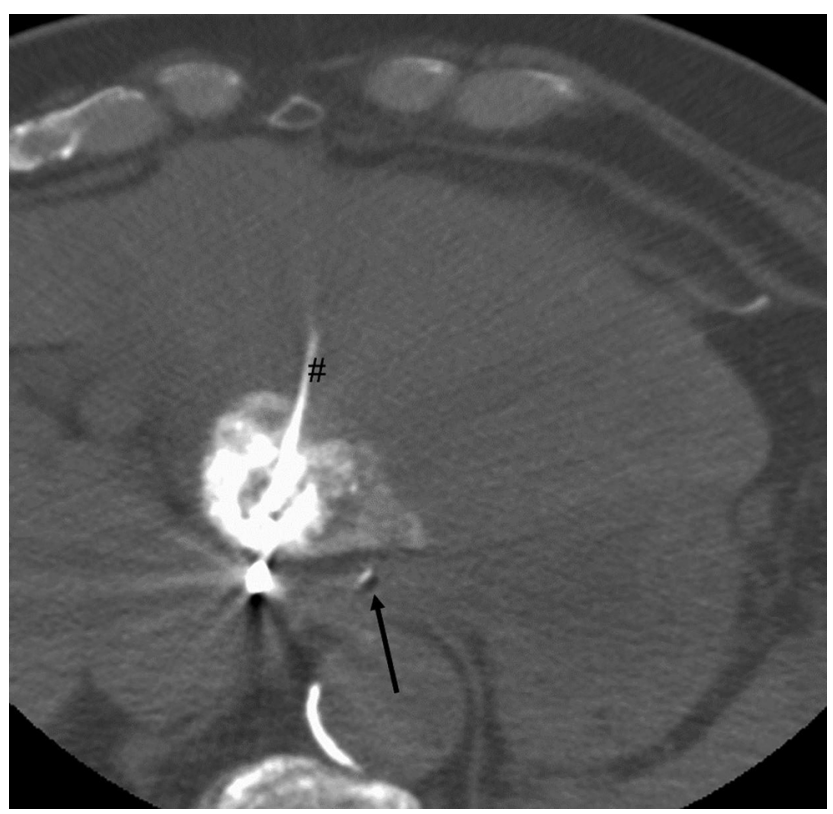

Fig. 3 After confirmation of the proper placement of the antenna (\#) on intraprocedural computed tomography, ablation is conducted with continual irrigation of cooled saline and aspiration via the nasal feeding tube (arrow) during the ablation.

in segment I. Following multidisciplinary discussions, we decided to perform percutaneous ablation for the deep lesion, followed by partial hepatectomy for the other metastases on the liver surface. The target tumor was interposed between the lower esophagus and the inferior vena cava and just below the confluence of the left- and middle hepatic veins ( - Fig. 5). Although MWA would have been a suitable 


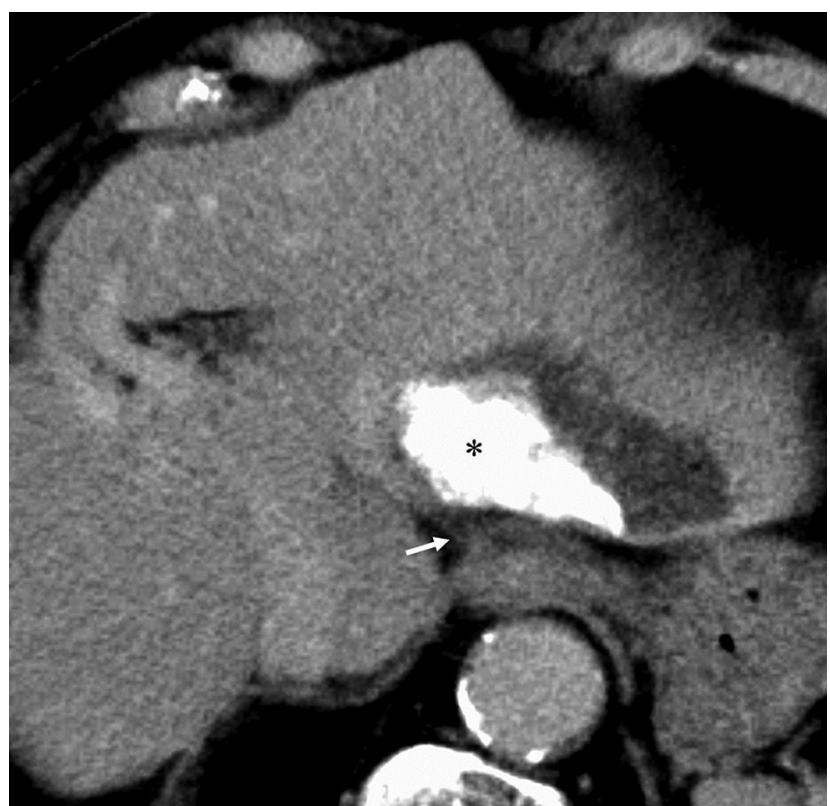

Fig. 4 Contrast-enhanced computed tomography 14 days later shows no thermal injury to the adjacent lower esophagus despite opacification of the fat (white arrow) around the tumor $\left({ }^{*}\right)$, indicating that the ablated area reaches the anterior esophageal wall.

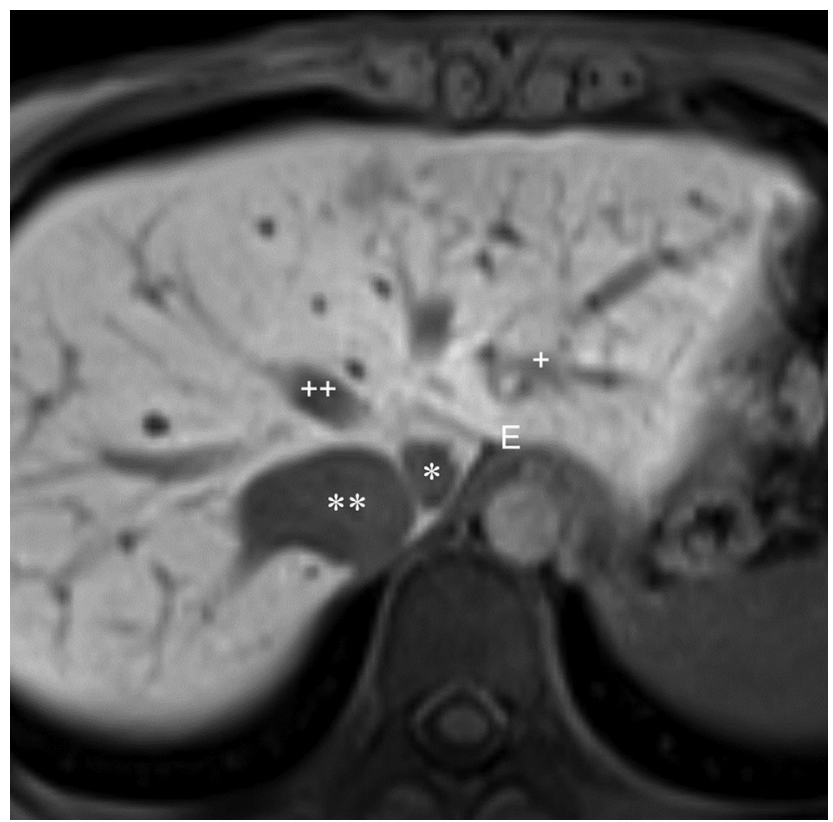

Fig. 5 T1-weighted magnetic resonance imaging depicts a $12-\mathrm{mm}$ metastatic tumor $\left({ }^{*}\right)$ in segment I, adjacent to the lower esophagus (E) and the inferior vena cava $\left({ }^{* *}\right)$, and just below the confluence of middle hepatic vein $(++)$ and left hepatic vein $\left(^{+}\right)$.

treatment because of its lower heat-sink effect from the adjacent vasculatures, it was not available at our institution at the time. Accordingly, three occlusion balloon catheters were inflated in each vessel, via the jugular or the femoral vein approaches, to reduce the heat-sink effect from each vessel and a 12-French nasal feeding tube was inserted into the lower esophagus in preparation for RFA using the Cool-tip RF ablation System E Series (Covidien Japan) under intravenous sedation with diazepam and pethidine administration and local anesthesia with lidocaine. A single electrode with a 2-cm active tip was inserted under fluoroscopic guidance, using the balloons that had been inflated with diluted contrast media for reference ( - Fig. 6). After CT verification of positioning the electrode in the desired portion of the tumor ( - Fig. 7), ablation was started at $40 \mathrm{~W}$. The

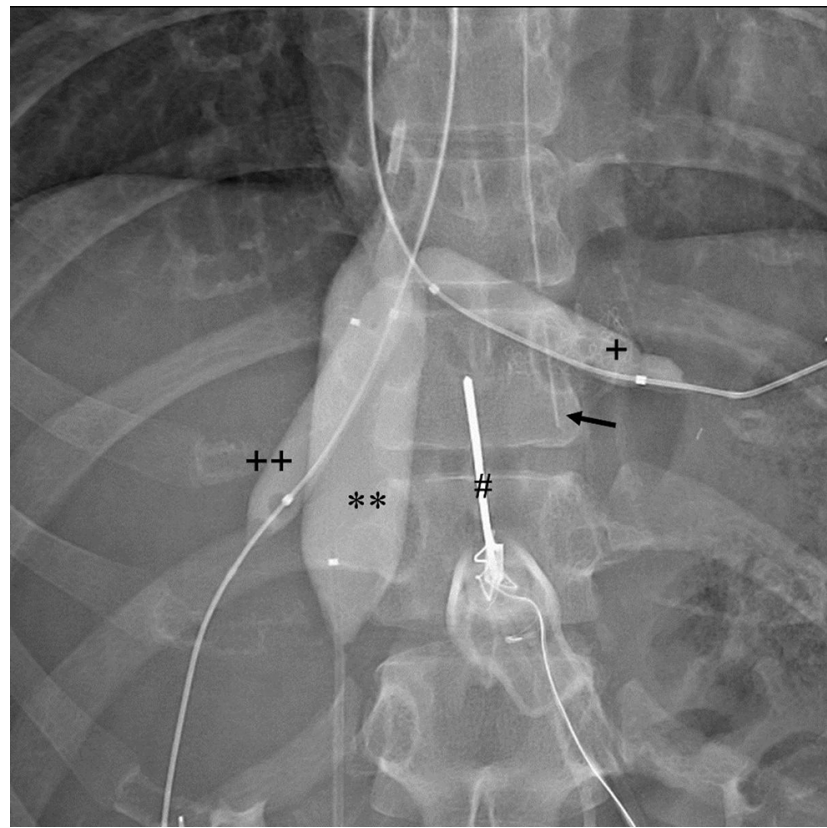

Fig. 6 A radiofrequency ablation electrode (\#) is inserted after inflation of three balloon-catheters placed in the inferior vena cava $\left({ }^{* *}\right)$, middle hepatic vein $(++)$, and left hepatic vein $(+)$. Note a nasal feeding tube (arrow) placed in the lower esophagus.

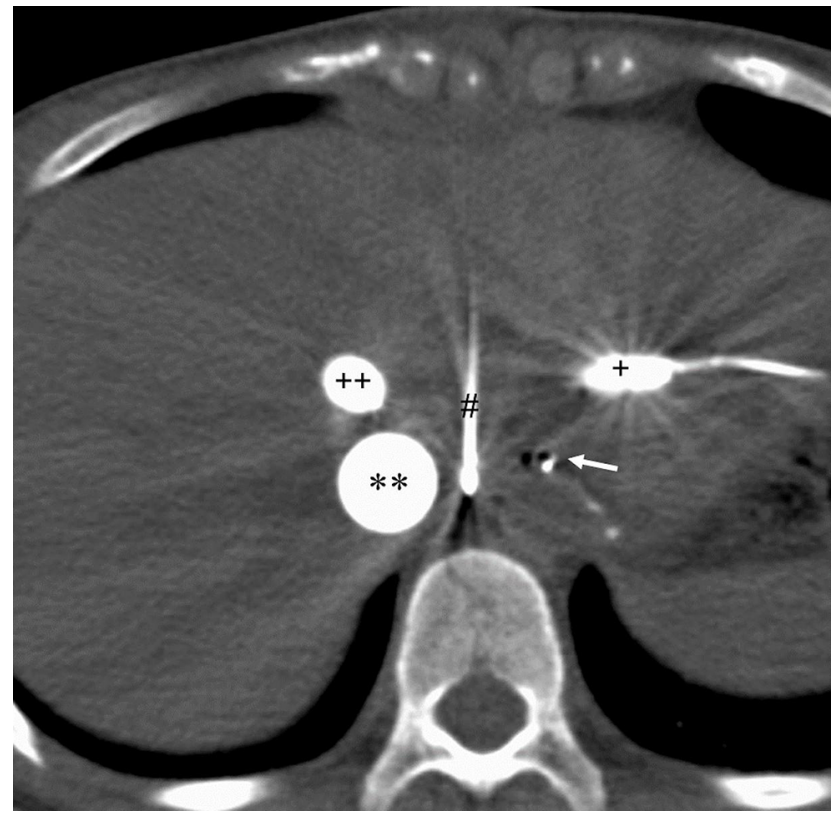

Fig. 7 After the correct electrode placement (\#) confirmed on computed tomography, ablation is initiated with intraluminal esophageal cooling via the tube (white arrow). Note the balloons in inferior vena cava $\left({ }^{* *}\right)$, middle hepatic vein $(++)$, and left hepatic vein $(+)$. 
power was increased by $10 \mathrm{~W}$ every minute, and roll-off occurred at $90 \mathrm{~W}$; subsequently, repeated ablation was conducted to obtain additional roll-off. The total ablation time was 7.5 minutes, according to our standardized ablation protocol. Similarly, the lower esophagus was cooled to prevent thermal injury during the procedure, as performed in Case 1. The patient felt mild fatigue with a low-grade fever until the next day and improved spontaneously. Contrast-enhanced CT obtained 2 days after the procedure showed complete tumor ablation with no esophageal injury or the occlusion of the adjacent vessels ( $\mathbf{- F i g . ~ 8 )}$ ). The patient was discharged 2 days after the procedure.

Following additional RFA for another liver metastasis in segment IV, partial hepatectomy was performed for the remaining metastases as planned. The patient has experienced no local recurrence or delayed esophageal complications for 3 years after the first ablation.

\section{Discussion}

Endoluminal cooling of the lower esophagus, by irrigation with cooled saline via a nasal feeding tube, is a simple and less invasive thermoprotective technique compared with conventional hydrodissection. In hydrodissection, a needle is precisely inserted around the lower esophagus via a transhepatic approach, which is further complicated by hepatic excursion and patient's respiratory movement. Furthermore, there is an increased risk of bleeding and radiation exposure

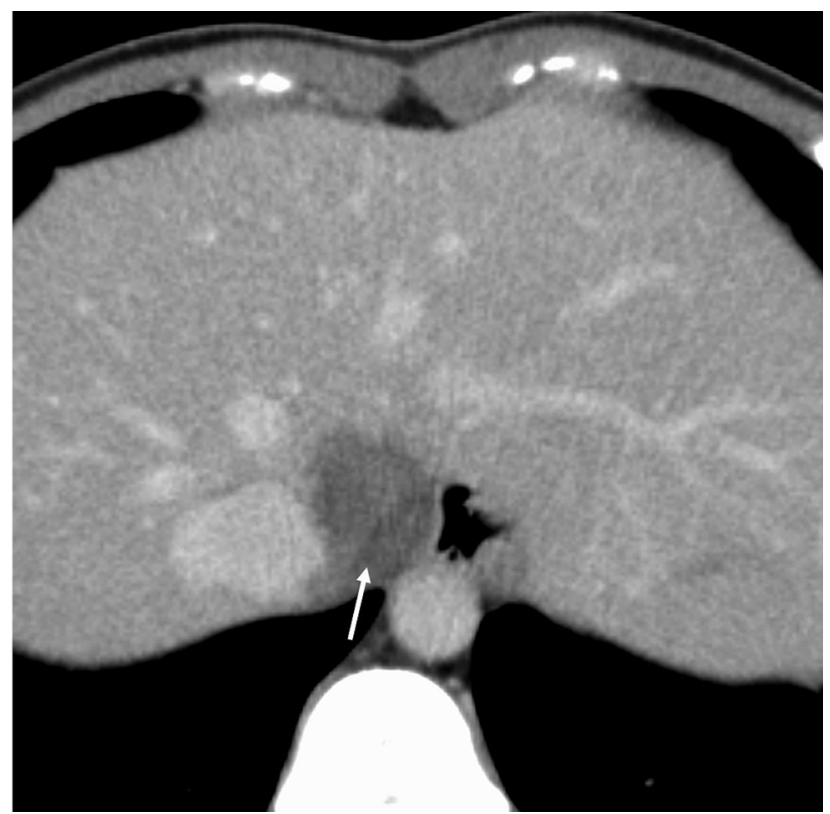

Fig. 8 Contrast-enhanced computed tomography obtained two days later shows that the tumor is completely encompassed by the ablated area (white arrow) with no thermal injury to the abutting esophageal wall. if the needle is advanced under CT-guidance, and the distribution of the injected fluid in a stable manner around the esophagus remains controversial.

In conclusion, these two cases indicate that continuous irrigation by cooled saline via a nasal feeding tube placed in the lower esophagus enables effective ablation of a juxtaesophageal tumor while reducing the risk of esophageal thermal injury. The actual risk of esophageal thermal injury and the necessity of its protection during ablation therapy for liver neoplasm remains controversial. Similarly, the effectiveness of esophageal cooling, which has previously been applied as a preventive measure for lethal atrioesophageal fistula, remains uncertain in atrial fibrillation ablation procedures. ${ }^{6,7}$ However, we believe that the method should be attempted for ablation procedures of the liver due to its simplicity and apparent effectiveness. Therefore, further evaluations should be performed to fully establish the feasibility of this thermoprotection.

\section{Informed Consent}

Informed consent was obtained from all individual participants for the procedures.

\section{Source of Support}

None.

\section{Conflict of Interest}

None.

\section{References}

1 Tateishi R, Shiina S, Teratani T, et al. Percutaneous radiofrequency ablation for hepatocellular carcinoma. An analysis of 1000 cases. Cancer 2005;103(6):1201-1209

2 Liang P, Wang Y, Yu X, Dong B. Malignant liver tumors: treatment with percutaneous microwave ablation-complications among cohort of 1136 patients. Radiology 2009;251(3):933-940

3 Koda M, Murawaki Y, Hirooka Y, et al. Complications of radiofrequency ablation for hepatocellular carcinoma in a multicenter study: an analysis of 16346 treated nodules in 13283 patients. Hepatol Res 2012;42(11):1058-1064

4 Garnon J, Cazzato RL, Caudrelier J, et al. Adjunctive thermoprotection during percutaneous thermal ablation procedures: review of current techniques. Cardiovasc Intervent Radiol 2019;42(3):344-357

5 Yamane T, Imai K, Umezaki N, et al. Perforation of the esophagus due to thermal injury after laparoscopic radiofrequency ablation for hepatocellular carcinoma: a case for caution. Surg Case Rep 2018;4(1):127

6 Kuwahara T, Takahashi A, Okubo K, et al. Oesophageal cooling with ice water does not reduce the incidence of oesophageal lesions complicating catheter ablation of atrial fibrillation: randomized controlled study. Europace 2014;16(6):834-839

7 Han HC, Ha FJ, Sanders P, et al. Atrioesophageal fistula: clinical presentation, procedural characteristics, diagnostic investigations, and treatment outcomes. Circ Arrhythm Electrophysiol 2017;10(11):e005579 